\title{
Cluster Head Selection Protocol using Fuzzy Logic for Wireless Sensor Networks
}

\author{
Sachin Gajjar \\ Institute of Technology, \\ Nirma University, \\ Ahmedabad, Gujarat, India.
}

\author{
Mohanchur Sarkar \\ Space Application Centre, \\ Indian Space Research \\ Organisation, \\ Ahmedabad, Gujarat, India
}

\author{
Kankar Dasgupta \\ Indian Institute of Space \\ Science and Technology, \\ Thiruvananthapuram, India
}

\begin{abstract}
Recent trends in field of wireless networks is setting up Wireless Sensor Networks that, senses specified parameter(s) related to environment; processes sensed data and wirelessly communicates it to a base station. Such networks open up a whole new range of applications, including precision agriculture, monitoring and tracking vehicles, animals and humans, battle-field surveillance, civil structural monitoring etc. All these applications require extended network lifetime, scalability, and traffic balancing among nodes in the network. Clustering is one of the effectual techniques for achieving these requirements. In clustering, geographically adjacent nodes are organized into virtual groups called clusters. One of the cluster node acts as a cluster head and rest as cluster members. This paper presents Cluster Head selection protocol using Fuzzy Logic (CHUFL). It uses node's parameters like: residual energy, reachability from its neighborhood, quality of communication link with its neighborhood and distance from base station as fuzzy input variables for cluster head selection. A comparative analysis of CHUFL with cluster head selection mechanism using fuzzy logic by Indranil et. al.; Cluster Head Election mechanism using Fuzzy logic (CHEF) by Kim et. al. and cluster head selection method for wireless sensor networks based on fuzzy logic by J. Anno et. al. shows that CHUFL is up to $20 \%$ more energy efficient and sends $72 \%$ more packets to base station compared to protocol by J. Anno et. al., one of the energy efficient clustering protocol.
\end{abstract}

\section{General Terms}

Wireless sensor network protocol design; algorithms; simulation; performance evaluation; comparative analysis

\section{Keywords}

Wireless sensor networks; clustering; cluster head selection; fuzzy logic

\section{INTRODUCTION}

The proliferation in Micro Electro Mechanical Systems (MEMS) technology for the development of smart sensors and advances in wireless communication technologies have geared growth in the field of Wireless Sensor Networks (WSN). Nodes in WSN are densely deployed in hostile environments where they may technically fail, die due to lack of power, be physically damaged or may face environmental interference. Further, nodes have severely limited computational, storage and power capabilities [1]-[2]. This demands energy efficient techniques to be used at all the stages of WSN protocol design. Clustering is one of techniques to prolong the network lifetime. In a clustering protocol the geographically adjacent nodes are organized into virtual groups called "clusters". One of the nodes is selected as a cluster head and rest in its neighborhood as cluster members. Clustering offers following advantages: (i) reduces collision during intra-cluster communication by coordinating media access mechanism of its cluster members (ii) offers load balancing by rotating cluster head (ii) reduces amount of information updates required (node deaths and joins in a cluster, need to be updated only by their cluster members) (ii) offers scalability and spatial reuse (non-neighbor clusters may use same frequency or code for transmission). In clustering protocol, cluster head works as a local coordinator for its cluster and does following: (i) arranges intra-cluster transmission schedule (ii) collects data from its cluster members (ii) combines several correlated data signals into a smaller set of information (data aggregation) (iii) forwards data to Base Station (BS). Several computational intelligence techniques like fuzzy logic, neural networks, reinforcement learning, swarm intelligence, evolutionary algorithms, artificial immune systems and reinforce learning have been proposed for cluster head selection in WSN [3]. From among these techniques, fuzzy logic is one of the best problem-solving control system methodologies that provides a simple way to arrive at a definite conclusion with imprecise, non-numerical, noisy, or missing input information. It uses heuristic knowledge and human reasoning to deal with contradictory situations and imprecise data. The capability of fuzzy logic is exploited in technical fields like: image, speech and signal processing; aerospace, robotics and embedded systems industries; along with non-technical fields like business, sales and marketing [4]. For WSN protocol design fuzzy logic offers following advantages: (i) the terms used in WSN protocol's performance ("lower latency", "longer lifetime") and the transmission media characteristics ("less noisy", "more busy") make their fuzzy representation easy and realistic. (ii) it can easily and efficiently deal with various uncertainties of WSNs, such as unreliable media, unpredictable changes in network topology and still arrive at a definite conclusion. (iii) fuzzy logic is flexible, scalable, fault tolerant, requires less system development cost, resources (computation and memory requirements) and design time. Hence, for WSN it is used in clustering, location updating [5], routing [6], improving accuracy of event detection [7], security [8], QoS support [9]. To this end, this paper presents Cluster Head selection protocol using Fuzzy Logic (CHUFL). It uses node parameters like (i) its residual energy (to efficiently run data aggregation and forward the cluster head data), (ii) its reachability from the neighboring nodes (to decrease the intra-cluster communication cost) and (iii) the quality of its communication link with its neighboring nodes (to increase the reliability of the algorithm) and (iv) distance 
of node from BS (to decrease inter-cluster communication cost) as fuzzy input variables for cluster head selection.

The remainder of paper is organized as follows: Section 2 discusses related work, Section 3 discusses CHUFL operation in detail, Section 4 provides comparative analysis of CHUFL with three well referred fuzzy based clustering protocols. Finally, the paper is concluded in Section 5.

\section{RELATED WORK}

A variety of clustering protocols have been proposed in the literature for selecting the cluster heads and organizing the clusters [10]. LEACH, the most popular architecture elects cluster heads based on probability model taking care that a node becomes a cluster head in round $\mathrm{r}$, only if it has not been a cluster head for previous (r-1) rounds [11]. Clustering with rotating cluster heads evenly distributes load among nodes. Since each node probabilistically decides whether or not to become cluster head, there might be cases when selected cluster heads are in close vicinity of each other or may be having less residual energy sacrificing overall performance of the network. Position-based Aggregator Node Election is a position-based clustering routing protocol [12]. Clustering is pre-determined before deployment of the network, and each node is pre-loaded with geographical information of its cluster. At start of each epoch, nodes in each cluster calculate a reference point depending on epoch number in a distributed manner. Then after, nodes in the cluster elect node that is closest to the reference point as cluster head for given epoch. Data aggregation in PANEL significantly reduces number of transmissions and receptions. However, PANEL assumes that clustering is pre-determined before deployment of the network and hence cannot adapt to dynamics of WSN. Algorithm for Cluster Establishment (ACE) allows a node to evaluate its potential as a cluster head before becoming one and give up if it is not best cluster head at any instance of time [13]. When a node decides to become a cluster head, it sends cluster head advertisement to its neighbors. In response, neighboring nodes becomes its follower. Each cluster head periodically checks potential of its followers to determine best candidate for new cluster head. It then gives up if one of its followers has more followers than it does. Migration of cluster heads helps in network repairs and makes the protocol robust. But it requires a large number of control packets to be send. In Base-Station Controlled Dynamic Clustering Protocol cluster formation technique takes care that each cluster head has an equal number of cluster members to balance network load and uniform distribution of cluster head in the network [14]. BS receives energy information of nodes, calculates average and chooses nodes with energy greater than average energy as cluster heads. BS then computes number of clusters and starts clustering. BS has global information of position and energy of nodes, so it can produce better clusters that require less energy for data transmission. But, being a centralized protocol makes it non-scalable and a single point failure protocol. Although all these protocols provide a valuable and strong contribution in WSN clustering, none of them uses computational intelligence. Clustering protocol by Indrail et. al. uses fuzzy logic with input variables as node's energy, concentration (number of nodes in neighborhood) and centrality (how much node is at center of cluster) for cluster head selection [15]. Fuzzy logic selects best cluster head in terms of node's energy and intra-cluster transmission cost. However, each node has to send information about its current location and energy level to BS in each round which is a transmission overhead. Cluster Head Election mechanism using Fuzzy Logic (CHEF) protocol overcomes this overhead by running fuzzy logic with residual energy of node and local distance (sum of distances between particular node and its neighbors within a specified radius) as fuzzy descriptors for cluster head selection at the node [16]. On the downside, CHEF doesn't consider inter-cluster communication cost for cluster head selection. Cluster Head Election protocol by Junpei Anno et. al. uses fuzzy logic with distance of cluster centroid from BS, residual energy of node and network traffic as inputs for cluster head selection [17]. The probability of the node to become cluster head is zero if its residual energy is less than a predefined value. The work does not suggests as to where the fuzzy logic will run and how the input fuzzy descriptors will be collected.

\section{CHUFL OPERATION}

The operation of CHUFL consists of: Neighbor finding and Steady state phases.

\subsection{Neighbor finding}

During this phase, each node broadcasts an INFO_MESG (containing its ID, location information) using a nonpersistent CSMA MAC protocol [18] to reach nodes in its transmission range. Each node stores the details of its neighboring nodes in its neighbor details table.

\subsection{Steady state}

It is divided into rounds. Each round begins with cluster head selection followed by clustering, data gathering at cluster head and finally data transfer to BS. A flowchart of operation of CHUFL is shown in Figure 1.

\subsubsection{Cluster Head Selection}

Nodes make independent decisions for becoming cluster heads using fuzzy logic without any central control as discussed below.

Fuzzy Inference System (FIS) design for cluster head selection: Mamdani Model is used for developing FIS for cluster head selection since it is simple, has widespread acceptance and is well suited to imprecise inputs [19]. FIS design steps are discussed next.

Fuzzification of inputs and outputs:

Input variables:

Residual Energy (indicated as RESIDUAL_ENERGY): For node to become a cluster head it should have more RESIDUAL_ENERGY compared to its neighboring nodes.

Node's reachability (indicated as REACHABILITY): It is measure of how much the node is reachable to its neighbor nodes within its transmission range. Reachability $r(i)$ of a node $i$ is defined as,

$$
r(i)=\frac{1}{N}\left(\sum_{j=1}^{j=N-1} d_{i j}\right)
$$

where $N$ is number of nodes in the transmission range (found during the neighbor finding), $d_{i j}$ is distance between node $i$ and $j$. For a node to become a cluster head it should have more number of neighboring nodes and hence a lower value of REACHABILITY.

Link Quality Indicator/distance of node from BS (indicated by LQI/DISTANCE): Link Quality Indicator (LQI) characterizes the quality reception of a packet at a node. It is precisely defined in 802.15.4 standard [20] and can be easily estimated by radio chips like CC2500 [21]. LQI is divided by distance of node from BS. With constant message length, deterioration in quality of reception of a packet is marked with decreased LQI. Further, a lesser distance of node from BS ensures energy savings in communication to BS [18]. Thus for a node to become a cluster head it should have a high LQI and should be at a lesser distance from the BS.

Output variable: 
POTENTIAL (indicated by POTENTIAL): It is a node's ability to become a cluster head. A large value of POTENTIAL indicates more ability of a node to become a cluster head.

The linguistic variables used to represent RESIDUAL_ENERGY, REACHABILITY and LQI/DISTANCE are divided into three levels: low, medium and high. The linguistic variable POTENTIAL is divided into seven levels: very small, small, rather small, medium, rather large, large, and very large.

Defining membership functions: Triangle and trapezoidal membership functions are used since their degree can be easily determined [19]. Triangle membership functions are used to represent the fuzzy input sets medium and trapezoid membership functions to represent low and high fuzzy sets. Similarly, triangle membership functions are used to represent output sets small, rather small, medium, rather large, large and trapezoid membership functions to represent very small and very large fuzzy sets.

The membership functions developed in Matlab [23] for the network scenario simulated in this paper are represented in Figures 1 to 4 .

Application of fuzzy operators and fuzzy rule evaluation: With 3 input variables and 3 levels for each, there are $3^{3}=27$ possible combinations for Rule base. Table 1 shows the rule base defined using "if then" rules with "and" operators among input variables.

Aggregation of all outputs: For particular values of 3 inputs there will be multiple rules fired. According to degree of membership for all rules fired, the outputs are unified. The maximum region covered for the output value is taken. Defuzzification: The aggregate of a fuzzy set encompasses a range of output values, and so must be defuzzified in order to resolve a single output value from the set. Centroid method is used for defuzzification.

Each node calculates POTENTIAL value using fuzzy if-then rule discussed above. If POTENTIAL > T \% (Threshold value between 1 and 100) then node becomes a COMPETITOR node for being a cluster head. Value of $\mathrm{T}$ must be selected such that it guarantees enough COMPETITOR nodes for quality cluster head selection and also results in less control message overhead. It is set to $40 \%$ for the simulations done in the paper. Each node then uses a non-persistent CSMA MAC protocol to advertise a COMPETITOR_MESG (containing its POTENTIAL, ID, location) to reach nodes within its transmission range. Node with highest value of POTENTIAL becomes cluster head.

\subsubsection{Clustering algorithm}

Nodes that have been selected to be cluster head broadcasts CLUS HEAD ADV MESG (containing node's ID) using a non-persistent CSMA $\mathrm{MAC}$ protocol within its transmission range that they are cluster heads for current round by setting it's transmit power. Each non-cluster head node then selects its cluster head as one for which received signal strength (RSSI) of CLUS_HEAD_ADV_MESG is largest assuming it to be closet cluster head. If there are ties, cluster head with least ID is chosen among tied ones. Each node then informs its cluster head that it wants to join its cluster with JOIN_CLUS_MESG (containing its ID, cluster head's ID). The cluster head sets up a TDMA schedule for data transmissions for its cluster members and transmits it to its cluster members. The TDMA schedule is set up such that the cluster member having highest POTENTIAL value is the first in the schedule. At end of first round, nodes in network know their fellow cluster members and their POTENTIAL. From next round onwards clusters are fixed for certain number of upcoming rounds (depending on the network conditions) and cluster head nodes are rotated among cluster members as per order of slots in TDMA schedule. Thus cluster advertisement and join messages need not be sent for each round. This saves time and energy of nodes during each round. Rotation of cluster head equally distributes energy load among all nodes in network.

\subsubsection{Data gathering at cluster head}

Once TDMA schedule is known to all cluster nodes data gathering operation starts. It is broken into frames. The cluster members send their data packets to cluster head (with transmit power enough to reach its cluster head) once per frame during their allocated transmission slot to reduce collisions. Its radio is turned off for rest of the time to save energy. On receiving data packets, cluster head performs data aggregation and aggregated data packets are relayed to BS through several cluster heads during data transfer to BS.

\subsubsection{Data transfer to the BS}

Data transmission to BS is carried out using dijkstra's shortest path routing algorithm through cluster heads in a multihop manner [22].

\section{SIMULATION AND ANALYSIS OF CHUFL}

Performance of CHUFL is compared with protocol by Indranil et. al.; CHEF and protocol by Anno et.al. Simulation parameters are shown in the Table 2. Figure 5 shows total residual energy of all the nodes in the network for first 260 rounds. It is maximum for CHUFL because of the following reasons: (i) it chooses cluster head which are having large number of neighbors which decreases energy required for intra cluster communication (ii) it chooses cluster head which are near the BS which decreasing energy required for inter cluster communication cost (iii) it reduces control transmission overheads (cluster advertisement and join messages need) by static clustering for specific number of rounds. Thus overall average energy consumption of each node is least in CHUFL and hence its residual energy is high compared to other protocols. Protocol by Indranil et. al. requires nodes to send its parameters to $\mathrm{BS}$ in each round increasing energy consumption of the nodes. CHEF and protocol by Anno et.al. do not consider the inter-cluster communication cost for cluster head selection which increases their energy consumption. Thus as seen in the Figure 6 and 7 total dead nodes for first 260 rounds is minimum in CHUFL and network lifetime performance in terms of first node dies (FND), half of the nodes alive (HNA) and last node dies (LND) is best for CHUFL. Figure 8 shows aggregated data received at BS is maximum in CHUFL. This is because CHUFL uses LQI in cluster head selection which sets up reliable communication link and increases number of packets received at BS. As shown in Table 3, throughput and goodput achieved by CHUFL is maximum amongst all protocol configurations. The netwok settling time and end-to-end latency for CHEF is minimum. This is because it uses only 2 node parameters as inputs to fuzzy system and its rule base table has 9 entries for cluster head selection which takes less compution time compared to all the other protocols (which use 3 fuzzy inputs and have 27 entries in their rule base table).

\section{CONCLUSION}

Protocols for WSN require to be energy efficient and reliable. These features led to design of CHUFL, a clustering protocol which minimizes energy consumption of cluster head by choosing cluster heads nearer to BS, having more residual energy and neighbors. Reliability of the protocol is increased by choosing cluster heads with a high value of LQI. Simulation results show that CHUFL provides best 
performance under tight energy, memory and computational constraints of the nodes and constraints of wireless transmission media compared to protocol by Indranil et.al., $\mathrm{CHEF}$ and protocol by Anno et. al.

\section{REFERENCES}

[1] Sachin Gajjar, Nilav Choksi, Mohanchur Sarkar, and Kankar Dasgupta, "Comparative analysis of Wireless Sensor Network motes", Proc. of International Conference on Signal Processing and Integrated Networks, pp. 426 - 431, 2014.

[2] S.H. Gajjar, S.N. Pradhan, and K.S. Dasgupta, "Wireless Sensor Networks: Application led research perspective", Proc. of IEEE Recent Advances in Intelligent Computational Systems, pp. 025 - 030, 2011.

[3] Raghavendra V. Kulkarni, Anna Forster, and Ganesh Kumar Venayagamoorthy, "Computational Intelligence in Wireless Sensor Networks: A Survey," IEEE Communications Surveys and Tutorials, vol. 13, no. 1, pp.68-96, 2011.

[4] Zadeh L. A.,'Is there a need for fuzzy logic?", Proc. of Annual Meeting of North American on Fuzzy Information Processing Society, pp. 1-3, 2008.

[5] L.Chou and L. Gen-Chung, "Location management using fuzzy logic control for wireless networks", Proc. of International Conferences on Info-tech and Info-net, pp. 339 - 344, 2001

[6] S.Y. Chiang and J. L. Wang, "Routing analysis using fuzzy logic systems in wireless sensor networks", Proc. of In Knowledge-Based Intelligent Information and Engineering Systems, pp. 966-973, 2008.

[7] Q. Liang and L. Wang, "Event detection in wireless sensor networks using fuzzy logic system", Proc. of IEEE International Conference in Computational Intelligence for Homeland Security and Personal Safety, pp. 52-55, 2005.

[8] H. Lee and T. Cho, "Fuzzy logic based key disseminating in ubiquitous sensor networks", Proc. of 10th International Conference on Advanced Communication Technology, pp. 958-962, 2008.

[9] F. Xia, W. Zhao, Y. Sun, and Y.C. Tian, "Fuzzy Logic Control Based QoS Management in Wireless Sensor/Actuator Networks", Journal of Sensors, vol. 7, no. 12, pp. 3179-3191, 2007.

[10] Sachin Gajjar, Mohanchur Sarkar, and Kankar Dasgupta,"Performance Analysis of Clustering Protocols for Wireless Sensor Networks", International Journal of Electronics and Communication Engineering and Technology, vol. 4, no. 6, pp. 107-116, 2013.

[11] W. B. Heinzelman, A. P. Chandrakasan, and H. Balakrishnan, "An Application Specific Protocol Architecture for Wireless Microsensor Networks", IEEE Transactions on Wireless Communications, vol. 1, no. 4, pp. 660-670, 2002.

[12] L. Buttyan and P. Schaffer, "Panel: Position-based aggregator node election in wireless sensor networks", Proc. of IEEE International Conference on Mobile Ad hoc and Sensor Systems, pp. 1-9, 2007.

[13] Chan H. and Perrig A. "ACE: An Emergent Algorithm for Highly Uniform Cluster Formation", Proc. of 1st
European Workshop on Sensor Networks, pp. 154-171, 2004.

[14] Murugunathan, S.D., Ma, D.C.F., Bhasin, R.I., and Fapajuwo, A.O., "A Centralized Energy-Efficient Routing Protocol for Wireless Sensor Networks", Proc. of IEEE Radio Communication, pp. 8-13, 2005.

[15] I. Gupta, D. Riordan, S. Sampalli, "Cluster-head election using fuzzy logic for wireless sensor networks", in Proc. of Annual Communication Networks and Services Research Conference, pp. 255-260, 2005.

[16] J. M. Kim, S. H. Park, Y. J. Han, and T. M. Chung, "CHEF: cluster head election mechanism using fuzzy logic in wireless sensor networks", Proc. of International Conference on Advanced Communication Technology, pp. 654-659, 2008.

[17] J. Anno, L. Barolli, F. Xhafa, and A. Durresi, “A Cluster Head Selection Method for Wireless Sensor Networks Based on Fuzzy Logic", Proc. of IEEE TENCON, pp. 14, 2007.

[18] T. S. Rappaport,"Wireless Communications: Principles and Practice", 2nd Edition, Prentice Hall Publications, Englewood Cliffs, NJ, 2001.

[19] J S R Jang, C T Sun and E Mizutani, "Neuro-fuzzy and soft computing A Computational approach to learning and machine intelligence", Prentice Hall Publications, 2003.

[20] IEEE Standard 802.15.4,"Wireless LAN Medium Access Control (MAC) and Physical Layer (PHY) specifications for low-rate wireless personal area networks (WPANS)", [Online] Available: http://standards.ieee.org/develop/ project/ 802.11.15.4. html.

[21] Texas Instruments, CC2500 datasheet [Online] Available: http: //focus.ti.com/lit/ds/symlink/cc2500

[22] R.K. Ahuja, T.L. Magnanti, and J.B. Orlin, "Network Flows: Theory, Algorithms, and Applications", Prentice Hall Publications, Englewood Cliffs, NJ, 1993.

[23] MathWorks Documentation Center [Online Available] http://www.mathworks.in/help/matlab/

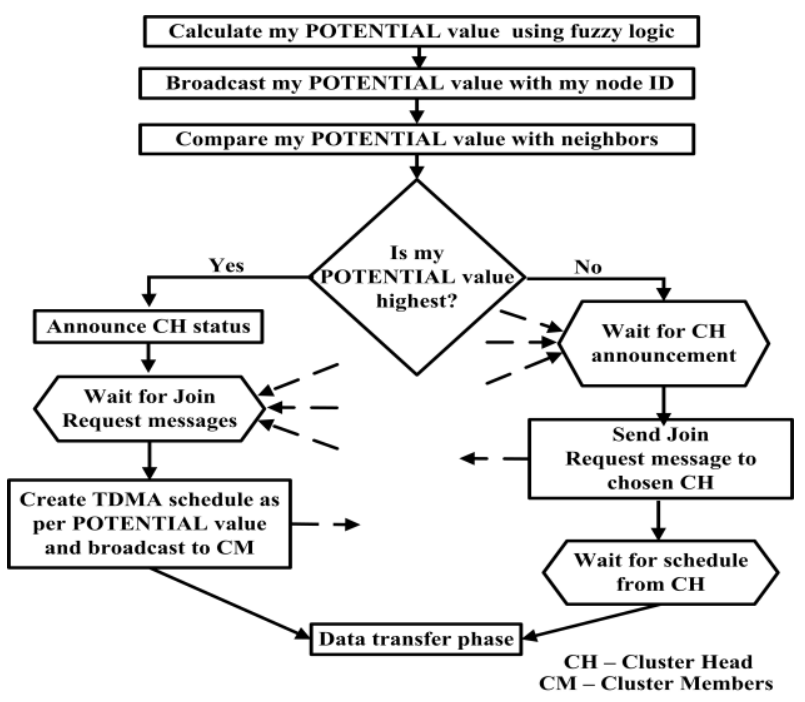

Fig 1: Flowchart of CHUFL operation 


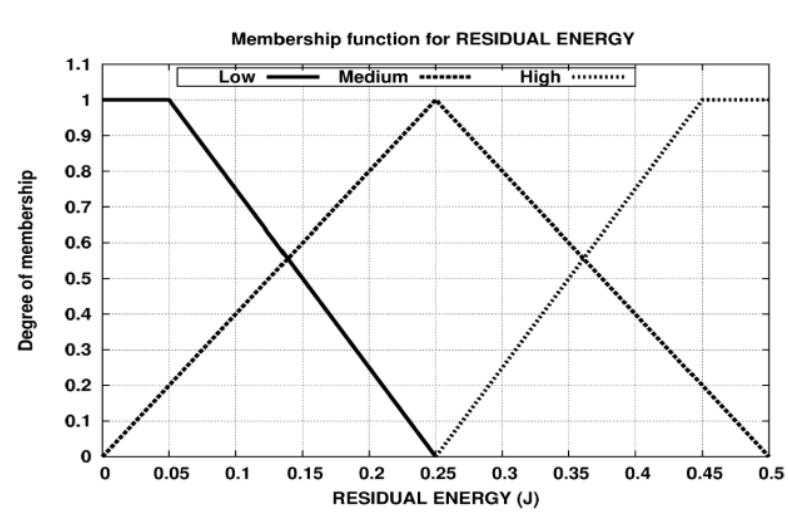

Fig 2: Fuzzy set for RESIDUAL_ENERGY

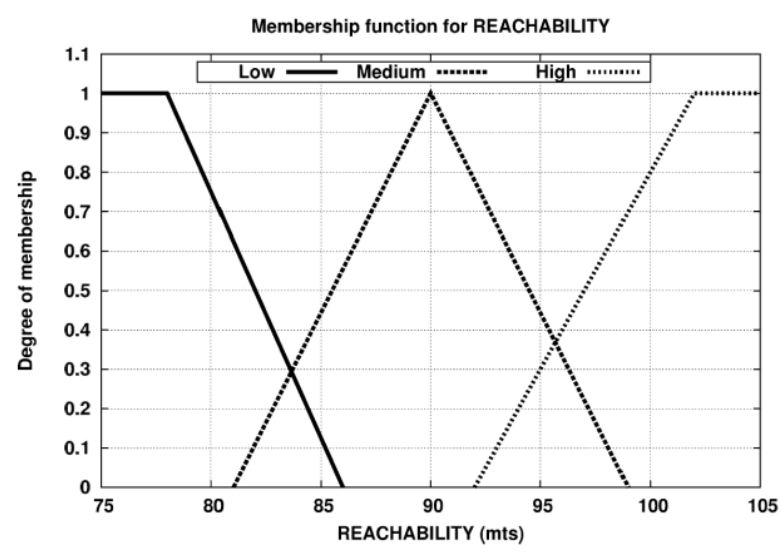

Fig 3: Fuzzy set for REACHABILITY

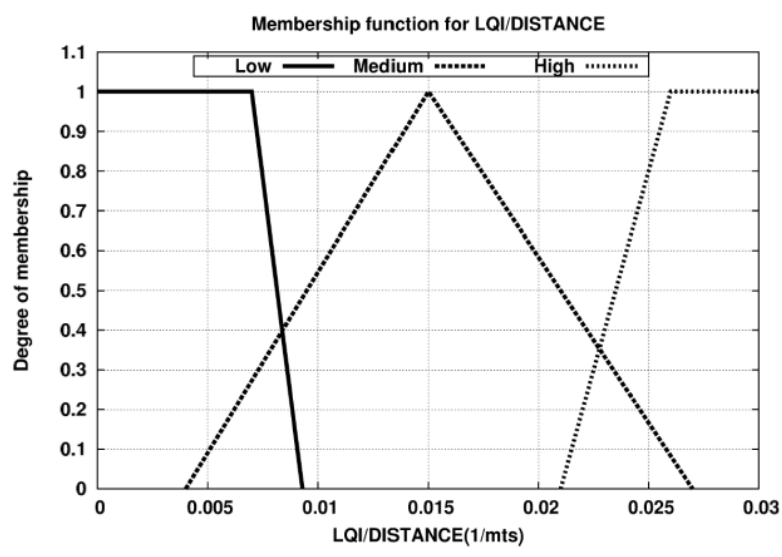

Fig 4: Fuzzy set for LQI/DISTANCE

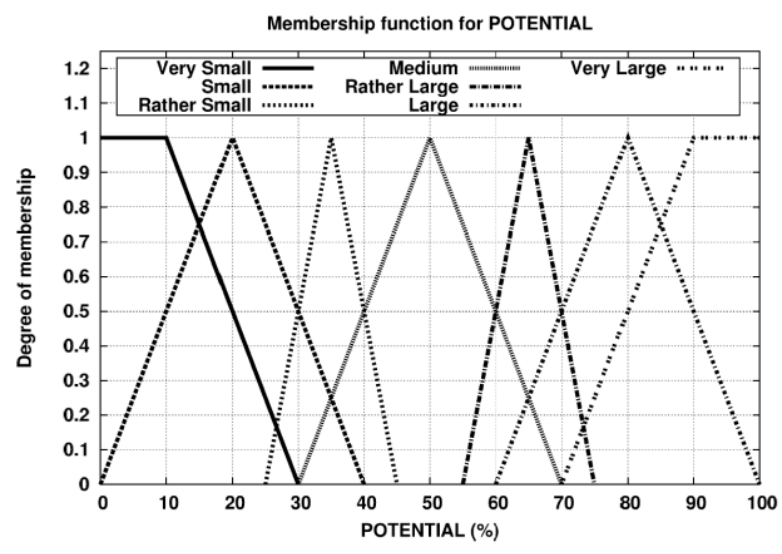

Fig 5: Fuzzy set for POTENTIAL

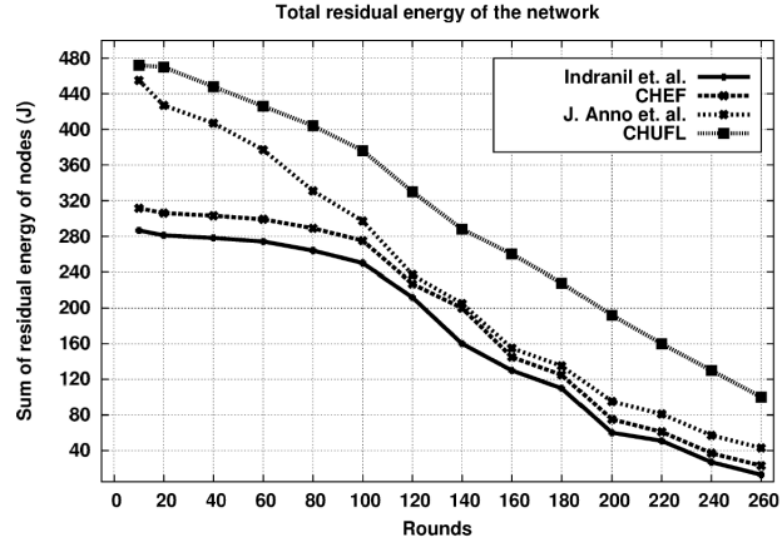

Fig 6: Total residual energy of the network

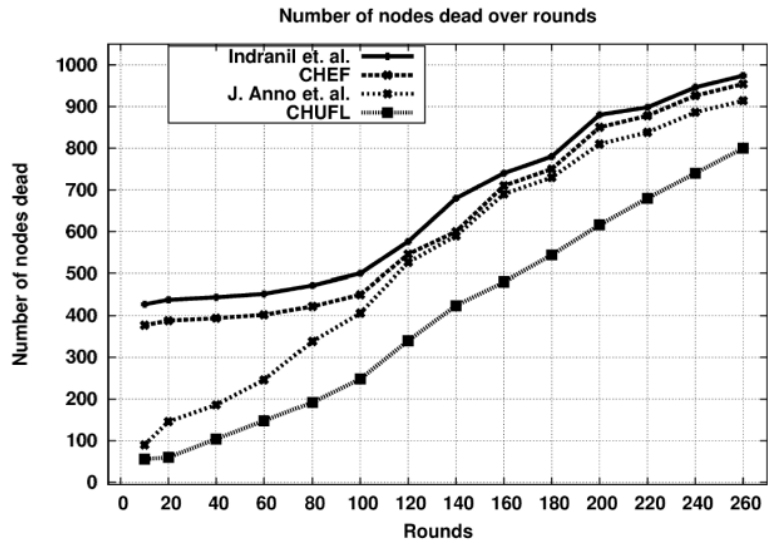

Fig 7: Number of dead nodes over rounds

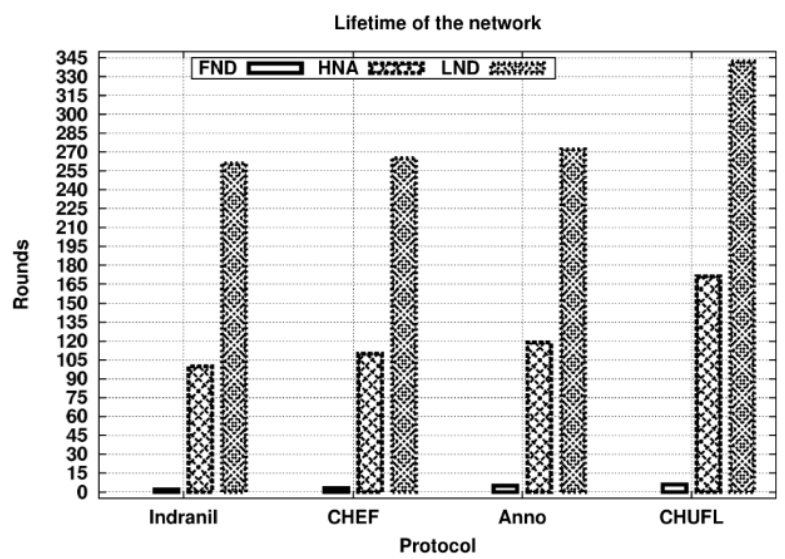

Fig 8: Time for FND, HNA and LND for all the protocols

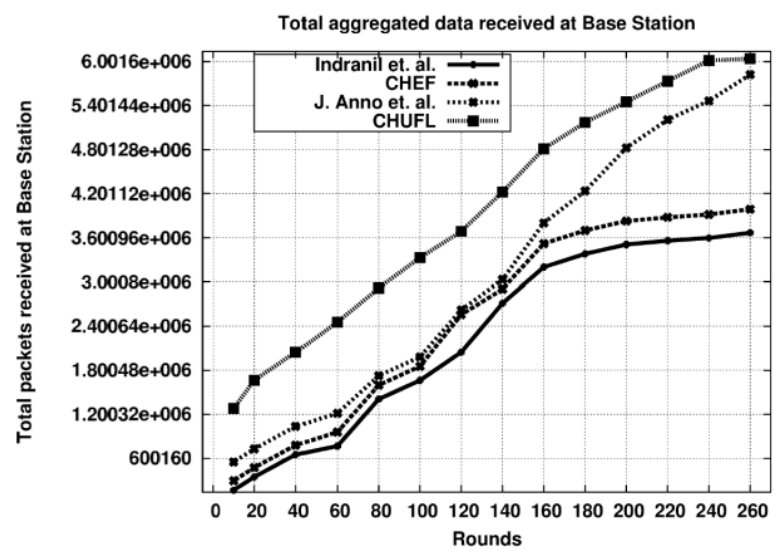

Fig 9: Aggregated data received at the Base Station 
Table 1. Fuzzy rule base for cluster head selection

\begin{tabular}{|c|c|c|c|c|}
\hline \multicolumn{5}{|c|}{$\begin{array}{c}\text { L=Low, M=Medium, H=High, } \text { S=Small, RS=Rather Small, VS=Very Small, } \\
\text { L=Large, } R L=\text { Rather Large, VL=Very Large }\end{array}$} \\
\hline \multirow[t]{2}{*}{ Rules } & \multicolumn{3}{|c|}{ Antecedents } & \multirow{2}{*}{$\begin{array}{l}\text { Consequents } \\
\text { POTENTIAL }\end{array}$} \\
\hline & RESIDUAL_ENERGY & RECHABILITY & LQI/DISTANCE & \\
\hline 1 & $\mathrm{~L}$ & $\mathrm{~L}$ & $\mathrm{~L}$ & $\mathrm{~S}$ \\
\hline 2 & $\mathrm{~L}$ & $\mathrm{~L}$ & $\bar{M}$ & $\mathrm{RS}$ \\
\hline 3 & $\mathrm{~L}$ & $\mathrm{~L}$ & $\mathrm{H}$ & $\mathrm{M}$ \\
\hline 4 & $\mathrm{~L}$ & $\bar{M}$ & $\mathrm{~L}$ & $\mathrm{~S}$ \\
\hline 5 & $\mathrm{~L}$ & $\mathrm{M}$ & $\mathbf{M}$ & RS \\
\hline 6 & $\mathrm{~L}$ & M & $\mathrm{H}$ & $\mathrm{M}$ \\
\hline 7 & $\mathrm{~L}$ & $\mathrm{H}$ & $\mathrm{L}$ & $\mathrm{VS}$ \\
\hline 8 & $\mathrm{~L}$ & $\mathrm{H}$ & $\mathbf{M}$ & $\mathrm{S}$ \\
\hline 9 & $\mathrm{~L}$ & $\mathrm{H}$ & $\mathrm{H}$ & $\mathrm{RS}$ \\
\hline 10 & $\mathrm{M}$ & $\mathrm{L}$ & $\mathrm{L}$ & $\mathrm{RL}$ \\
\hline 11 & $\mathrm{M}$ & $\mathrm{L}$ & $\mathbf{M}$ & $\mathrm{RL}$ \\
\hline 12 & $\mathrm{M}$ & $\mathrm{L}$ & $\mathrm{H}$ & $\mathrm{L}$ \\
\hline 13 & $\mathrm{M}$ & $\mathrm{M}$ & $\mathrm{L}$ & $\mathrm{L}$ \\
\hline 14 & $\mathrm{M}$ & $\mathbf{M}$ & $\mathbf{M}$ & RL \\
\hline 15 & $\mathrm{M}$ & $\mathrm{M}$ & $\mathrm{H}$ & $\mathrm{L}$ \\
\hline 16 & $\mathrm{M}$ & $\mathrm{H}$ & $\mathrm{L}$ & $\mathrm{RS}$ \\
\hline 17 & $\mathrm{M}$ & $\mathrm{H}$ & $\mathbf{M}$ & $\mathrm{M}$ \\
\hline 18 & $\mathbf{M}$ & $\mathrm{H}$ & $\mathrm{H}$ & $\mathrm{RL}$ \\
\hline 19 & $\mathrm{H}$ & $\mathrm{L}$ & $\mathrm{L}$ & RL \\
\hline 20 & $\mathrm{H}$ & $\mathrm{L}$ & $\mathrm{M}$ & $\mathrm{L}$ \\
\hline 21 & $\mathrm{H}$ & $\mathrm{L}$ & $\mathrm{H}$ & VL \\
\hline 22 & $\mathrm{H}$ & $\mathrm{M}$ & $\mathrm{L}$ & $\mathrm{L}$ \\
\hline 23 & $\mathrm{H}$ & $\mathrm{M}$ & $\mathbf{M}$ & RL \\
\hline 24 & $\mathrm{H}$ & $M$ & $\mathrm{H}$ & $\mathrm{L}$ \\
\hline 25 & $\mathrm{H}$ & $\mathrm{H}$ & $\mathrm{L}$ & $\mathrm{M}$ \\
\hline 26 & $\mathrm{H}$ & $\mathrm{H}$ & $\mathbf{M}$ & RL \\
\hline 27 & $\mathrm{H}$ & $\mathrm{H}$ & $\mathrm{H}$ & $\mathrm{L}$ \\
\hline
\end{tabular}

Table 2. Simulation settings

\begin{tabular}{|l|l|}
\hline Parameter & Description \\
\hline Nodes & 1000 \\
\hline Network size & $1000 \mathrm{~m} \mathrm{x} 1000 \mathrm{~m}$ \\
\hline BS location & $(0,0)$ \\
\hline Data size & 6400 bytes \\
\hline Control packet & $100 \mathrm{bits}$ \\
\hline Initial energy & $0.5 \mathrm{~J}$ \\
\hline Energy consumption model & As described in $[11]$ \\
\hline Radio amplifier energy & $10 \mathrm{pJ} / \mathrm{bit} / \mathrm{m}^{2}$ \\
\hline Radio electronics energy & $50 \mathrm{~nJ} / \mathrm{bit}$ \\
\hline Data aggregation energy & $5 \mathrm{nj} / \mathrm{bit} / \mathrm{signal}^{2}$ \\
\hline Simulator & $\mathrm{Matlab}[23]$ \\
\hline
\end{tabular}

Table 3. Simulation Results

\begin{tabular}{|l|c|c|c|c|}
\hline $\begin{array}{l}\text { Protocol } \\
\text { configuration }\end{array}$ & $\begin{array}{c}\text { Throughput } \\
(\boldsymbol{\%})\end{array}$ & $\begin{array}{c}\text { Goodput } \\
(\mathbf{\%})\end{array}$ & $\begin{array}{c}\text { Network settling } \\
\text { time (secs) }\end{array}$ & $\begin{array}{c}\text { Latency } \\
\text { (secs) }\end{array}$ \\
\hline Indranil et. al. & 42 & 91 & 10 & 0.4 \\
\hline CHEF & 46 & 93 & 7 & 0.2 \\
\hline Anno et. al. & 67 & 92 & 8 & 0.3 \\
\hline CHUFL & 72 & 95 & 9 & 0.3 \\
\hline
\end{tabular}

